\title{
Unusual Chromophore and Cross-Links in Ranasmurfin: A Blue Protein from the Foam Nests of a Tropical Frog**
}

\author{
Muse Oke, Rosalind Tan Yan Ching, Lester G. Carter, Kenneth A. Johnson, Huanting Liu, \\ Stephen A. McMahon, Malcolm F. White, Carlos Bloch, Jr., Catherine H. Botting, \\ Martin A. Walsh, Aishah A. Latiff, Malcolm W. Kennedy, Alan Cooper, * and James H. Naismith*
}

Ranasmurfin is an unusual blue protein isolated from the nests of a Malaysian tree frog, Polypedates leucomystax, ${ }^{[1]}$ showing the rich chemical diversity displayed by biomolecular foams. Many species of tropical frogs use foams to protect delicate eggs and developing embryos against environmental challenges. These nests act as miniature ecosystems containing a spectrum of novel proteins and other macromolecules with functions related to foam stabilization and adhesion, resistance to microbial degradation, predation, or dehydration, providing a biocompatible environment for embryonic development. This work forms part of our wider study of the intriguing physical and chemical properties of biofoams as unusual examples of biological soft matter. ${ }^{[2]}$

[*] Dr. R. T. Y. Ching, ${ }^{[+]}$Prof. A. Cooper

WestChem Department of Chemistry

University of Glasgow, Glasgow G12 8QQ (UK)

Fax: (+ 44) 141-330-2910

E-mail: alanc@chem.gla.ac.uk

Dr. M. Oke, ${ }^{[+]}$Dr. L. G. Carter, Dr. K. A. Johnson, Dr. H. Liu,

Dr. S. A. McMahon, Prof. M. F. White, Dr. C. H. Botting,

Prof. J. H. Naismith

Centre for Biomolecular Science and

The Scottish Structural Proteomics Facility

The University, St Andrews KY16 9ST (UK)

Fax: (+44) 1334-467-229

E-mail: naismith@st-and.ac.uk

Prof. M. W. Kennedy

Division of Environmental and Evolutionary Biology

Institute of Biomedical and Life Sciences

University of Glasgow, Glasgow G12 8QQ (UK)

Dr. C. Bloch, Jr.

Embrapa Recursos Genéticos e Biotecnologia

Parque Estação Biológica

Asa Norte-Brasilia, 70910-900 (Brazil)

Dr. M. A. Walsh

Medical Research Council France

European Synchrotron Radiation Facility

F-38043, Grenoble CEDEX (France)

Prof. A. A. Latiff

Doping Control Centre Penang, Universiti Sains Malaysia

11800 Minden, Penang (Malaysia)

['] These authors contributed equally to this work.

[**] We thank the BBSRC, the Wellcome Trust, and the Scottish Higher Education Funding Council (SHEFC) for financial support and Dr. Neeta Sinnappah, Jeet Sukamaran, Norsham Yaakob, Aw Ai Fong, Lim Ming Sang, and Lim Tau Chin for help with fieldwork and sample collection.

() Supporting information for this article is available on the WWW under http://dx.doi.org/10.1002/anie.200802901.
P. leucomystax is an abundant Rhacophorid frog that is widespread in Malaysia and surrounding regions of SouthEast Asia. During mating, the female produces a protein-rich fluid that she, together with the attending male, whips up into a sticky foam nest incorporating the fertilized eggs (see the Supporting Information Figure S1). The nests are usually attached to vegetation or other structures overhanging water into which the tadpoles fall when fully developed (usually after 3-4 days in the wild). ${ }^{[3]}$ These nests exhibit a variety of pigmentation, usually pale creamy white/orange when first formed, but frequently develop a dark blue/green color with time (hours to days). Gel electrophoresis of natural foam nest material shows that this pigmentation co-migrates with an approximately $26 \mathrm{kDa}$ protein, designated ranasmurfin (PlRSF-1) ${ }^{[1]}$ clearly identifiable as a blue/green band in the electrophoresis gel, even under denaturing conditions and without staining. UV/Vis spectra of the purified protein show that the color derives from absorbance in the 500-700 nm region, peaking around 680-700 nm (Figure 1), together with typical $280 \mathrm{~nm}$ protein absorbance and subsidiary bands around $360 \mathrm{~nm}$. This blue coloration is remarkably stable and persists even after protein denaturation (with urea or sodium dodecylsulfate) or partial proteolysis (trypsin). The color is unaffected by treatment with $\mathrm{NaBH}_{4}$, cyanoborohydride, hydroxylamine, or ascorbate. The chromophore shows reversible spectral shifts with $\mathrm{pH}$ (Figure 1), which are consistent with the presence of two ionizable moieties with $\mathrm{p} K_{\mathrm{a}}$ values of 6 and 9 , and the color fades slowly (overnight)

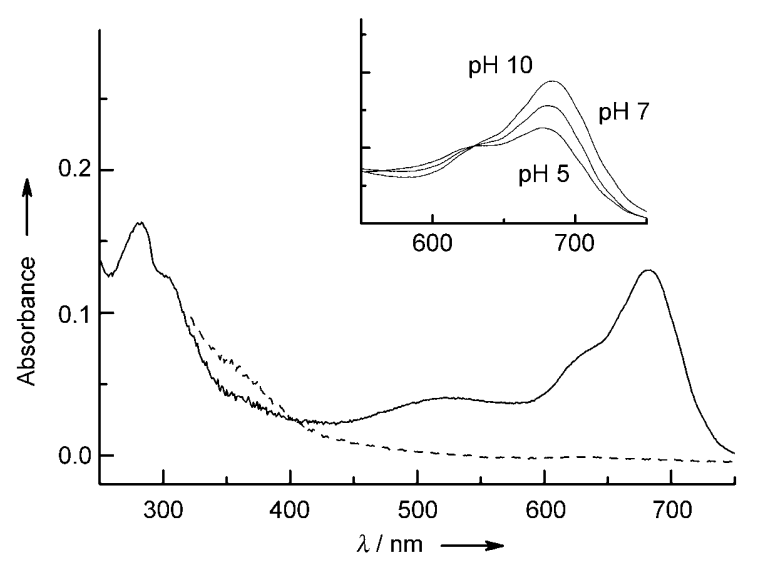

Figure 1. UV/Vis absorbance spectrum of ranasmurfin at neutral $\mathrm{pH}$ (solid line) and after treatment with $0.25 \mathrm{~mm}$ NBS (dashed line). Inset: $\mathrm{pH}$ dependence of PI-RSF-1 spectrum. 
upon treatment with ethylenediaminetetraacetic acid (EDTA) and dithiothreitol (DTT; Figure S9 in the Supporting Information). The chromophore is bleached rapidly (seconds) by low concentrations $(0.25 \mathrm{~mm})$ of $N$-bromosuccinimide (NBS, Figure 1).

Ranasmurfin, purified from natural material, crystallizes readily as deep blue crystals that diffract well, allowing protein structure determination to $1.16 \AA$ resolution (Figures 2 and 3; see the Supporting Information for crystallographic details). Initial X-ray diffraction experiments indicated the presence of a heavy metal in the protein, which was subsequently identified as zinc by X-ray fluorescence of the crystals (Ref. [1] and Supporting Information). Zinc was also

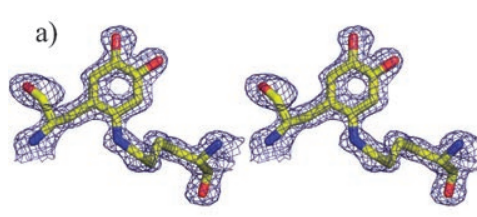

c)

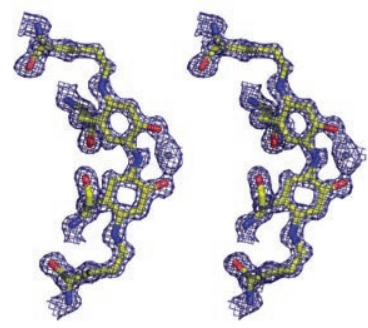

b)

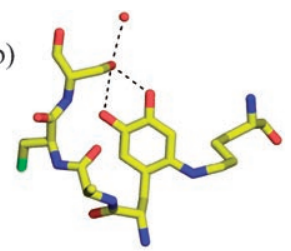

d)

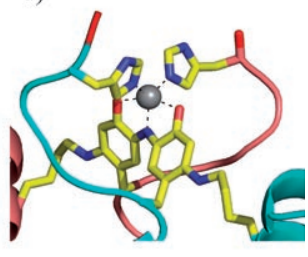

Figure 2. Protein-derived modifications in PI-RSF-1; $C$ yellow, $\mathrm{O}$ red, $\mathrm{N}$ blue, $S$ green. a) Stereo view of the electron density map of the Tyr2Lys 31 LTQ cross-link computed by using coefficients $2 F_{o}-F_{c}\left(F_{o}\right.$ and $F_{c}$ are the observed and calculated structure factors, respectively). Map contours were drawn at $1.5 \sigma$ of the final model. b) LTQ (monomer) environment in PI-RSF-1 showing hydrogen bonding to an adjacent Ser residue and a water molecule (red sphere). c) Stereo view of the electron density map of bis(LTQ) showing continuous electron density for covalent link between chains A and B. d) Coordination of $\mathrm{Zn}^{2+}$ (gray sphere) at the dimer interface by bis(LTQ) and the His 112 residue from each monomer.

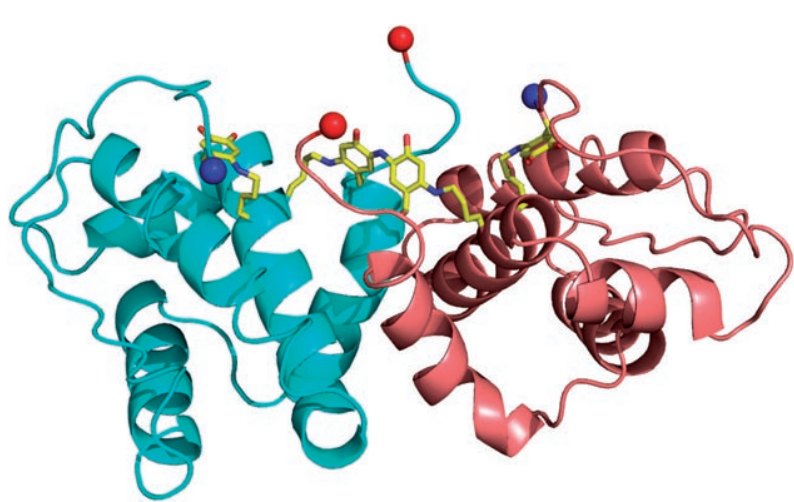

Figure 3. Crystal structure of the PI-RSF-1 dimer. Monomers A and B are colored cyan and salmon, respectively. Covalent cross-links formed by the four-residue inter-subunit linkage (bis(LTQ)) and intra-subunit two-residue cross-link (LTQ) are shown in stick representation. C yellow, $\mathrm{O}$ red, $\mathrm{N}$ blue, disulfide bonds green. Blue and red spheres represent the $\mathrm{N}$ and $\mathrm{C}$ termini, respectively. the dominant metal detected by ICP-OES experiments on purified protein solutions (ca. $1.2 \mathrm{~mol} \mathrm{Zn} / 26 \mathrm{kDa}$ ). The presence of a heavy metal facilitated the structure determination. The phases from the $\mathrm{Zn}$ atom extended to $1.6 \AA$ and gave a well-resolved electron-density map in which two molecules per asymmetric unit could be traced. Phases were extended to $1.16 \AA$ by solvent flattening and twofold averaging. This resolution was sufficient to identify most amino acids in the polypeptide chain, even in the absence of the complete protein or encoding cDNA sequence, and minor ambiguities were resolved by comparison with peptide sequences determined de novo by mass spectrometry. This procedure revealed the protein to be a $2 \times 13 \mathrm{kDa}$ dimer with an amino acid sequence (see the Supporting Information) unlike any in current databases, thus confirming the novelty of this protein.

Even in the very first map we could see clear density for several cross-links, some of which were obviously disulfides, but others appeared to involve aromatic rings. As the refinement progressed, these features were identified as bis(LTQ) and LTQ modifications (described below), which were only included in the last stages of refinement when the electron density was unambiguous. Experimental structure and phases have been deposited with PDB code $2 \mathrm{VH} 3$.

Ranasmurfin is a covalently linked homodimer with 113 amino acids per monomer that is folded in a novel $\alpha$-helical motif and stabilized by a range of cross-links (Figures 2, 3, and the Supporting Information). Each subunit contains three internal disulfide bonds (Cys4-Cys62, Cys17-Cys65, Cys37Cys101) together with an unusual lysine tyrosyl quinone (LTQ) linkage between the side chains of Lys31 and Tyr2 (Figure 4). Posttranslational modifications of this kind have<smiles>[14CH3]Nc1cc(O)c(O)cc1CC[18F]</smiles>

LTQ
bis-LTQ

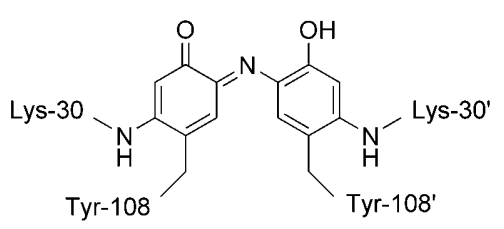

Figure 4. Intra-subunit (LTQ) and inter-subunit (bis (LTQ)) cross-links found in PI-RSF-1. Note that adjacent residues (Lys30, Lys31) are involved in these cross-links, adding further to the covalent stabilization of the protein structure (see also Figure $\mathrm{S} 4$ in the Supporting Information).

previously been described as cofactors or intermediates in (copper) lysyl oxidase enzyme mechanisms, ${ }^{[4-9]}$ but the crystallographic structure reported herein constitutes its first visualization as a stable entity in a native protein. Unlike the lysyl oxidase system, the LTQ group in Pl-RSF-1 is largely buried from solvent (Figure S5 in the Supporting Information) and has no coordinating metal. Instead, the two quinone oxygen atoms make a bifurcated hydrogen bond with the side chain of Ser-5, which is also hydrogen bonded to a water molecule (Figures $2 \mathrm{~b}$ and S5 in the Supporting Information).

Even more remarkable is the unprecedented four-residue (Lys-Tyr-N-Tyr-Lys) bis(LTQ) linkage between the two 
ranasmurfin subunits (Figures 2-4). This unusual structure, together with two His residues-one from each of the adjacent monomers-forms the binding site for the single zinc species (presumably $\mathrm{Zn}^{2+}$ ) that copurifies from nest material. The zinc is coordinated by two $\varepsilon$-nitrogen atoms (one from His112 in each chain) and by the tyrosyl hydroxy groups and linking atom of bis(LTQ), giving an approximately tetrahedral arrangement around the metal center. The planes of the two Tyr rings are rotated by about $27^{\circ}$ relative to each other. This extended aromatic system, involving the side chains of Lys30 and Tyr108 of one subunit linked through a (putative) nitrogen atom to the equivalent side chains in the adjacent subunit, provides both inter- and intra-subunit covalent stabilization of the protein structure, and is also most likely to be the blue chromophore. Analysis with PISA $^{[10]}$ gives a buried surface area of $970 \AA^{2}$ and less complementary packing than might normally be anticipated for a protein dimer. This result suggests that the dimer depends upon the covalent linkage for its integrity.

Although the identity of the bridging atom in the bis(LTQ) moiety cannot be determined unambiguously from the electron density alone $(\mathrm{C}, \mathrm{N}$, or $\mathrm{O}$ are all feasible at this resolution), nitrogen is the most likely on chemical grounds. Carbon would be an unlikely ligand for zinc, and an oxygen linkage would not allow sufficient electron delocalization to explain the blue pigmentation, and analogous diphenyl ethers are generally colorless. ${ }^{[11]}$ Nitrogen, however, would be a good ligand for the zinc, and comparable $\mathrm{N}$-linked analogues (such as indophenols and quinones ${ }^{[12-14]}$ ) are commonly highly colored, presumably because $\mathrm{sp}^{2}$ hybridization at the nitrogen atom allows extended conjugation between the aromatic rings. Such linkage is common in indophenol dyes, ${ }^{[14]}$ for which the addition of electron-donating groups ${ }^{[14]}$ and an increase in the dihedral angle between aromatic groups ${ }^{[15,16]}$ add further to the red shift in absorbance. Direct support for the assignment of nitrogen as the linking atom comes from model compound experiments on indophenol, solubilized in water as a cyclodextrin complex (see the Supporting Information for details). Like ranasmurfin, this simple N-linked biphenyl chromophore is sensitive to $\mathrm{pH}$ (Figure 5). It is similarly unaffected by treatment with borohydride, hydroxylamine, or ascorbate, but is rapidly bleached by NBS under the same conditions as for Pl-RSF-1 (cf. Figures 1 and 5).

This indophenol-like assignment is reinforced by fluorescence studies. Ranasmurfin shows UV fluorescence emission $\left(\lambda_{\mathrm{ex}}=280 \mathrm{~nm}, \lambda_{\mathrm{em}}=340 \mathrm{~nm}\right)$ that, like the absorbance, is sensitive to NBS (Figure 6). There is no detectable fluorescence emission in the visible region above $500 \mathrm{~nm}$. Such properties are normally considered typical of intrinsic tryptophan/protein fluorescence; NBS oxidation is used as a quantitative tool for measuring Trp content and reactivity. ${ }^{[17]}$ However, we find no Trp residues either in the de novo sequence or crystal structure of Pl-RSF-1, nor in preliminary 1D NMR spectra of the natural protein. This paradox is resolved by the observation (Figure 6) of very similar fluorescence and NBS reactivity for indophenol, suggesting that the protein (UV) fluorescence arises in this case from the bis(LTQ) subunit and that any similarities with Trp fluorescence are merely coincidental.

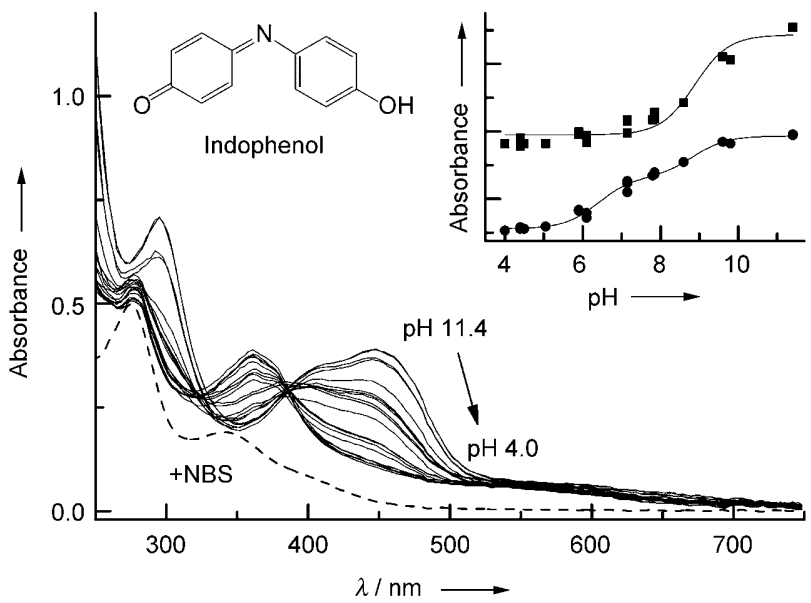

Figure 5. Absorbance spectra of indophenol: $\beta$-cyclodextrin complex in the $\mathrm{pH}$ range 4-11 (solid lines) and after treatment with $0.25 \mathrm{~mm}$ NBS, pH 7 (dashed line). Inset: $\mathrm{pH}$ dependence of indophenol absorbance at $295 \mathrm{~nm}$ (squares) and $448 \mathrm{~nm}$ (circles). The solid lines correspond to a theoretical titration for two groups with $\mathrm{p} K_{\mathrm{a}}$ values of 6.4 and 8.9 .
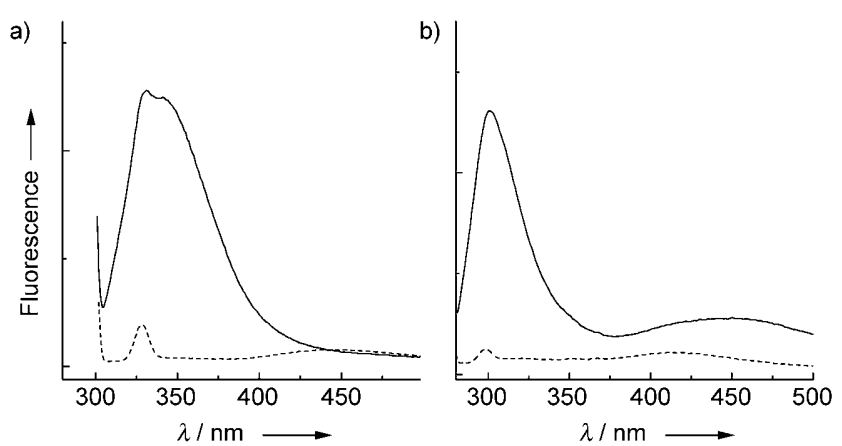

Figure 6. Fluorescence properties of PI-RSF-1 and indophenol. a) Intrinsic fluorescence emission $\left(\lambda_{\text {ex }}=295 \mathrm{~nm}\right.$ ) of PI-RSF-1 (solid line) and after treatment with NBS (dashed line). b) Intrinsic fluorescence emission $\left(\lambda_{\mathrm{ex}}=270 \mathrm{~nm}\right.$ ) of indophenol: $\beta$-cyclodextrin at $\mathrm{pH} 7$ (solid line) and after treatment with NBS (dashed line). Fluorescence spectra are uncorrected, and the small emission peaks at around $330 \mathrm{~nm}$ (a) or $300 \mathrm{~nm}$ (b) correspond to Raman scattering from solvent water.

We can only speculate at this stage about the biological function of ranasmurfin and the mechanism of its unusual posttranslational modifications, but its presence in the foam at relatively high levels suggests that it is involved, at least in part, in foam stabilization, adhesion, or other mechanical properties. Blue proteins are relatively rare in biology, and the chromophore of Pl-RSF-1 has little similarity with the polyenes, carotenoids, or porphyrins found in more familiar blue/green proteins. However, LTQ and related compounds are implicated as cofactors in protein cross-linking for matrix stabilization (connective tissues ${ }^{[5,6,8]}$ or dopamine-based protein adhesives and cements from mussels and other marine organisms $\left.{ }^{[18,19]}\right)$ and there are plausible mechanisms extending such schemes to obtain bis(LTQ) structures (see the Supporting Information). The blue coloration might thus be simply an adventitious by-product of such cross-linking 
reactions during foam maturation and stabilization, though it might also form part of camouflage or sunscreen mechanisms for photoprotection of the eggs and tadpoles in the highly oxygenated environment of the nests, which are frequently exposed to full tropical sunlight.

Biomolecular foams are now becoming more widely appreciated as an intriguing form of soft matter with a wide repertoire of biological functions. ${ }^{[2,20]}$ They are also rich sources of novel proteins. The work presented herein indicates that P1-RSF-1 is a new member of the quinoprotein family, ${ }^{[5]}$ and our demonstration of unusual cross-links in a naturally occurring protein, particularly the unforeseen 4residue bis(LTQ) chromophore, adds further to the growing family of posttranslational modifications that are difficult to predict from DNA sequence data alone. ${ }^{[4]}$

\section{Experimental Section}

Full details are given in the Supporting Information. Briefly, ranasmurfin was purified from natural foam nest material by gelfiltration and, after screening for optimal conditions, was crystallized by using a nanodrop crystallization robot. X-ray fluorescence and diffraction data were collected at the European Synchrotron Radiation Facility (ESRF, BM14) and the structure was solved by using MAD phasing from $\mathrm{Zn}$. Partial amino acid sequences were determined by mass spectrometry analysis of peptides after treatment with proteolytic enzymes. The protein was characterized in solution by using standard techniques (SDS-PAGE, UV/Vis, fluorescence spectroscopy) and tested for reactivity with a battery of standard protein modification reagents. Similar methods were used to compare the spectroscopy and reactivity of the model compound, indophenol, dispersed in the same aqueous buffers using $\beta$-cyclodextrin.

Received: June 18, 2008

Published online: September 9, 2008

Keywords: chromophores · cofactors · foam .

protein modifications $\cdot$ protein structures
[1] S. A. McMahon, M. A. Walsh, R. T. Y. Ching, L. G. Carter, M. Dorward, K. A. Johnson, H. T. Liu, M. Oke, C. Bloch, M. W. Kennedy, A. A. Latiff, A. Cooper, G. L. Taylor, M. F. White, J. H. Naismith, Acta Crystallogr. Sect. F 2006, 62, 1124.

[2] A. Cooper, M. W. Kennedy, R. I. Fleming, E. H. Wilson, H. Videler, D. L. Wokosin, T. J. Su, R. J. Green, J. R. Lu, Biophys. J. 2005, 88, 2114.

[3] C. D. Yorke, J. Herpetol. 1983, 17, 235.

[4] V. L. Davidson, Biochemistry 2007, 46, 5283.

[5] J. P. Klinman, Proc. Natl. Acad. Sci. USA 2001, 98, 14766.

[6] M. Mure, Acc. Chem. Res. 2004, 37, 131.

[7] M. Mure, S. X. Wang, J. P. Klinman, J. Am. Chem. Soc. 2003, 125, 6113.

[8] S. X. Wang, M. Mure, K. F. Medzihradszky, A. L. Burlingame, D. E. Brown, D. M. Dooley, A. J. Smith, H. M. Kagan, J. P. Klinman, Science 1996, 273, 1078.

[9] S. X. Wang, N. Nakamura, M. Mure, J. P. Klinman, J. SandersLoehr, J. Biol. Chem. 1997, 272, 28841.

[10] E. Krissinel, K. Henrick, Comput. Life Sci. Proc. 2005, 3695, 163.

[11] H. E. Ungnade, V. Kerr, E. Youse, Science 1951, 113, 601.

[12] H. Auterhoff, H. J. Pankow, Arch. Pharm. 1967, 300, 103.

[13] D. N. Kramer, R. M. Gamson, F. M. Miller, J. Org. Chem. 1959, $24,1742$.

[14] H. Zollinger, Color Chemistry: Syntheses, Properties, and Applications of Organic Dyes and Pigments, 3rd ed., Wiley$\mathrm{VCH}$, Zurich, 2003.

[15] M. Adachi, Y. Murata, S. Nakamura, J. Am. Chem. Soc. 1993, 115,4331

[16] M. Adachi, Y. Murata, S. Nakamura, J. Org. Chem. 1993, 58, 5238.

[17] T. F. Spande, B. Witkop, Methods Enzymol. 1967, 11, 498.

[18] R. J. Stewart, J. C. Weaver, D. E. Morse, J. H. Waite, J. Exp. Biol. 2004, 207, 4727.

[19] J. H. Waite, Integr. Comp. Biol. 2002, 42, 1172.

[20] J. C. Castilla, P. H. Manriquez, A. P. Delgado, L. Gargallo, A. Leiva, D. Radic, Proc. Natl. Acad. Sci. USA 2007, 104, 18120. 